\title{
Aire controls the expression of Prss29 in the thymus.
}

\author{
Shahan Mamoor ${ }^{1}$ \\ 1Thomas Jefferson School of Law \\ San Diego, CA 92101 \\ mamoorsk@tjsl.edu
}

Aire, the autoimmune regulator, promotes promiscuous transcription of tissue-restricted antigen in the thymus for negative selection $(1,2)$. By studying the transcriptomes of the thymuses of Aire knock-out mice using two independent published datasets $(3,4)$, we found that Prss29 was among the genes whose expression most changes between wild-type and Aire knock-out mice in the medullary epithelial cells of the thymus. The expression of Prss29 was significantly decreased in the absence of Aire. Prss29 is an implantation-specific protease that is important for the processes of embryonic invasion and implantation (5). We conclude that Aire controls the expression of Prss29 in the thymus and suggest that this may function in immunological privilege of the fetus.

Keywords: thymus, medullary epithelial cells, mTEC, differential gene expression, systems biology of the thymus, Prss29 


\section{Introduction}

Negative selection, the process by which developing lymphocytes that are reactive to self-antigens ((antigens expressed in the tissues of the body, or tissue-restricted antigens (TRA)) can be detected and deleted. The requires the presentation of TRA in the thymus, and the autoimmune regulator gene Aire functions in negative selection by promoting the expression of TRA in the medullary epithelial cells of the thymus (mTEC).

To understand the function of Aire, we studied the thymuses of Aire knock-out mice using two published datasets. We found that in the mTEC of mice that possessed a deletion in the Aire gene, Prss29 was among the genes whose expressed changed the most compared to mTEC from wild-type mice. Prss29 expression was almost absent in the mTEC of Aire knockout mice. We demonstrate control of Prss29, a serine protease expressed in the uterus and pre-implantation embryo, and suggest that it Aire promotes Prss29 expression for the purposes of immunologic tolerance at the maternal-fetal interface.

\section{Methods}

We used datasets GSE102527 (3) and GSE14365 (4) to perform this analysis, using GEO2R.

Koh et al. used Affymetrix Mouse Gene 2.0 ST Array to generate the GSE102527 microarray dataset.

Hubert et al. Affymetrix Mouse Genome 430 2.0 Array Array to generate the GSE14365 microarray dataset.

The "Benjamini and Hochberg" method of p-value adjustment was utilized for all analyses. Log-transformation was applied to all data. The NCBI category of platform annotation was utilized for all analyses. 
A unpaired, two-tailed t test was performed to evaluate the statistical significance of difference in Prss29 expression between Aire-sufficient and Aire-deficient genotypes using PRISM (8.1.2 (227)).

\section{Results}

We used whole transcriptome profiling to understand in an unbiased fashion the function of Aire at the transcriptional level in vivo. We found that mTEC isolated from Aire knock-out mice differentially expressed the serine protease Prss29.

Prss29 was the 42nd most differentially expressed gene when comparing the transcriptomes of mTEChi from Aire-deficient and wild-type mice. This was statistically significant (Table $1 ; \mathrm{p}=1.35 \mathrm{E}-05)$. In a separate dataset, Prss29 was the 16 th most differentially

expressed gene when comparing the transcriptomes of mTEChi from Aire-deficient and wild-type mice. This was also statistically significant (Table $1 ; p=1.01 \mathrm{E}-05)$.

We then extracted the exact mRNA expression values for Prss29 in the mTEC of both Aire-deficient and wild-type mice. Prss29 was expressed at significantly lower levels across both datasets in Aire knockout mice. In the absence of Aire, Prss29 expression in mTEC was almost ablated. This was statistically significant in both cases (Figure 1; $p=0.0005$ and Figure 2; $p=0.0072$, respectively). Thus, Prss29 is expressed at significantly lower levels in the mTEC of the thymuses of Aire-deficient mice than in the mTEC of wild-type mice.

\section{Discussion}

The thymus is the site of negative selection, the process whereby self-reactive developing lymphocytes are deleted from the T-cell repertoire in order to prevent autoimmune reactions against a multitude of self-antigens expressed in the periphery $(6,7)$. The Aire molecule is in large part responsible for initiating promiscuous expression of these tissuerestricted antigen for their presentation by the class II major histocompatibility complex on the 
surface of mTEC of the thymus $(2,3,8)$. Here, we studied the transcriptomes of mTECs from mice that possessed specific deletions in the Aire gene $(4,5)$. We found, by comparing mTEC transcriptomes from Aire-deficient and wild-type mice that the serine protease Prss29 was among the genes most differentially expressed in the thymuses of Aire-deficient mice. In the absence of Aire, Prss29 expression was almost lost. Thus, Aire controls the expression of Prss29 in the mTEC. This was a consistent finding across two independent datasets. Thus, we found that Prss29 was among the genes most differentially expressed in the mTEC of Airedeficient mice, and that Prss29 expression was significantly decreased and almost lost in Airedeficient mTEC.

Prss29 is an implantation-specific serine protease that is expressed in both the uterus and pre-implantation embryo (5). We suggest that Aire controls the expression of Prss29 in the mTEC of the thymus in order to present Prss29 antigen to developing lymphocytes to delete lymphocytes that would otherwise, once entering the periphery, go on to react with Prss29expressing cells of the uterus or the embryo during pregnancy. Thus, Aire may control Prss29 expression in mTEC for negative selection in order to support maternal-fetal tolerance. Future studies can specifically address the impact of Prss29 deletion in mTEC of the thymus on immunological privilege of the fetus. 


\section{References}

1. Liston, A., Lesage, S., Wilson, J., Peltonen, L. and Goodnow, C.C., 2003. Aire regulates negative selection of organ-specific T cells. Nature immunology, 4(4), pp.350-354.

2. Anderson, M.S., Venanzi, E.S., Chen, Z., Berzins, S.P., Benoist, C. and Mathis, D., 2005. The cellular mechanism of Aire control of T cell tolerance. Immunity, 23(2), pp.227-239.

3. Koh, A.S., Miller, E.L., Buenrostro, J.D., Moskowitz, D.M., Wang, J., Greenleaf, W.J., Chang, H.Y. and Crabtree, G.R., 2018. Rapid chromatin repression by Aire provides precise control of immune tolerance. Nature immunology, 19(2), pp.162-172.

4. Hubert, F.X., Kinkel, S.A., Crewther, P.E., Cannon, P.Z., Webster, K.E., Link, M., Uibo, R., O'Bryan, M.K., Meager, A., Forehan, S.P. and Smyth, G.K., 2009. Aire-deficient C57BL/6 mice mimicking the common human 13-base pair deletion mutation present with only a mild autoimmune phenotype. The Journal of Immunology, 182(6), pp.3902-3918.

5. Sharma, N., Liu, S., Tang, L., Irwin, J., Meng, G. and Rancourt, D.E., 2006. Implantation Serine Proteinases heterodimerize and are critical in hatching and implantation. BMC developmental biology, 6(1), p.61.

6. Surh, C.D. and Sprent, J., 1994. T-cell apoptosis detected in situ during positive and negative selection in the thymus. Nature, 372(6501), pp.100-103.

7. Kishimoto, H. and Sprent, J., 1997. Negative selection in the thymus includes semimature T cells. The Journal of experimental medicine, 185(2), pp.263-272.

8. Gray, D., Abramson, J., Benoist, C. and Mathis, D., 2007. Proliferative arrest and rapid turnover of thymic epithelial cells expressing Aire. The Journal of experimental medicine, 204(11), pp.2521-2528 
Table 1

\begin{tabular}{|r|l|l|l|l|l|l|}
\hline \multicolumn{1}{|l|}{ Rank ID } & p-value & t & B & logFC & gene \\
\hline GSE102527 & & & & & & \\
\hline $\mathbf{4 2}$ & 17334656 & $1.35 \mathrm{E}-05$ & $-1.4 \mathrm{E}+01$ & 3.866768 & -3.88 & Prss29 \\
\hline GSE14365 & & & & & & \\
\hline $\mathbf{1 6}$ & $1420282 \_$s_at & $1.01 \mathrm{E}-05$ & $-1.97 \mathrm{E}+01$ & 3.3939 & -7.10 & Prss29 \\
\hline
\end{tabular}

Table 1: Prss29 is one of the most differentially expressed genes in the Aire-deleted mTEC of the thymus.

The rank of differential expression, the transcript or probe ID, the p-value of differential expression, t, a moderated t statistic, B, the log-odds of differential expression between Airesufficient and Aire-deleted states, the log-fold change in expression between Aire-sufficient and Aire-deleted conditions, and the gene name is shown in this chart (all provided by GEO2R). 


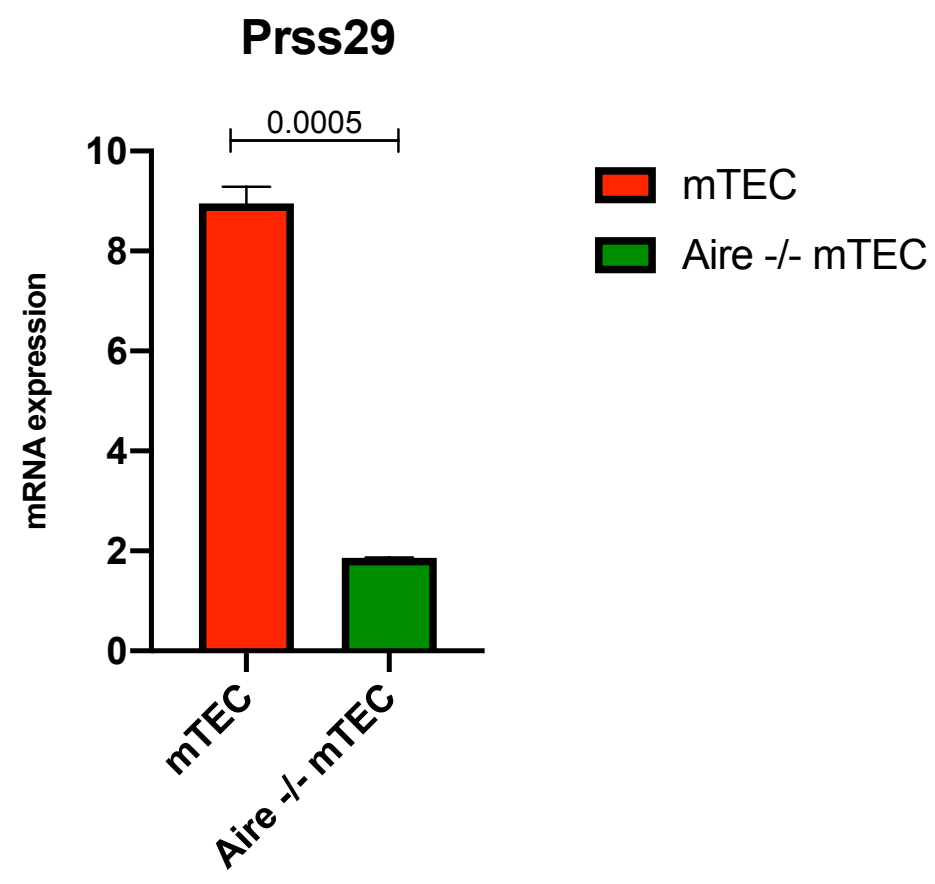

Figure 1: Prss29 is expressed at significantly higher levels in the mTEC of the thymus.

The mRNA expression level of Prss29 is shown in this bar graph, with the error bars denoting the standard error of the mean; $n=3$ for mTEC and $n=2$ for Aire -/- mTEC. A statistical test was performed to evaluate the significance of difference between Aire-sufficient and Aire-deleted conditions and the results of this test, a p-value, is shown on each graph. 


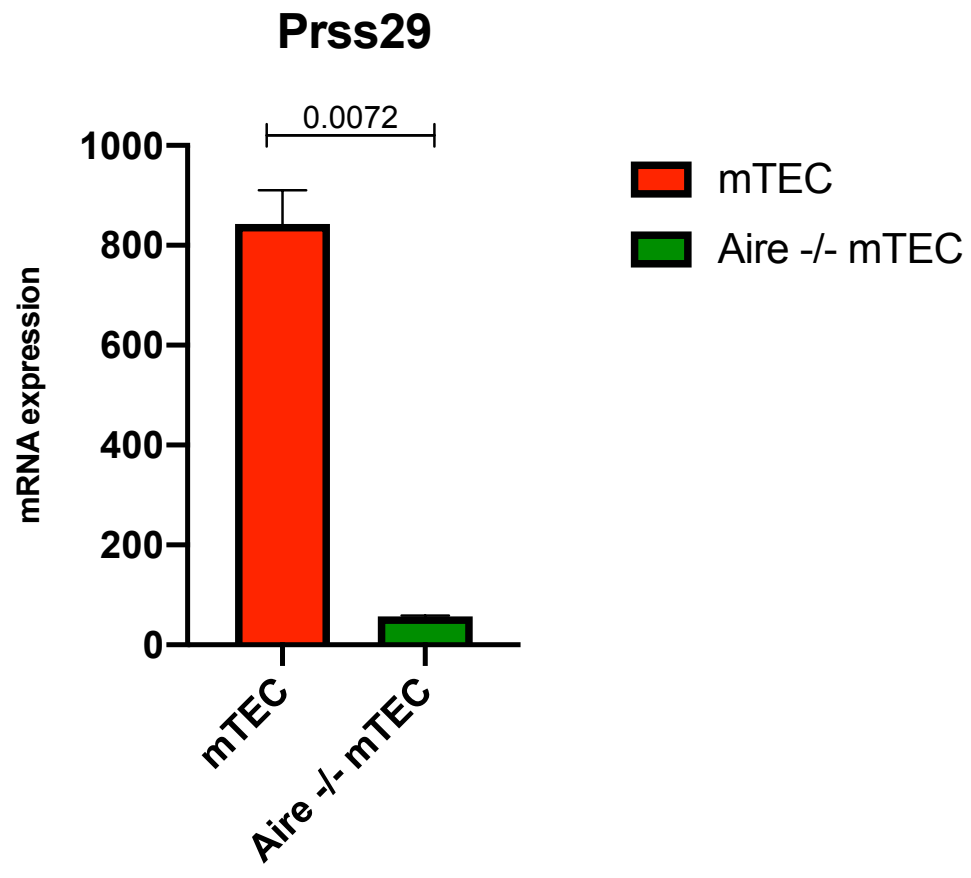

Figure 2: Prss29 is expressed at significantly higher levels in mTEChi of the thymus.

The mRNA expression level of Prss29 is shown in this bar graph, with the error bars denoting the standard error of the mean; $n=2$ for $\mathrm{mTEC}^{\text {hi }}$ and $n=2$ for Aire -/- mTEChi. A statistical test was performed to evaluate the significance of difference between Aire-sufficient and Airedeleted conditions and the results of this test, a p-value, is shown on each graph. 